\title{
Comparison of Muscle-Tendon Interaction of Human M. Gastrocnemius Between Ankle- and Drop-Jumping
}

\author{
Senshi Fukashiro*, Sadao Kurokawa ${ }^{* *}$, Dean C. Hay $^{* *}$ and Akinori Nagano ${ }^{* * *}$ \\ * Graduate School of Interdisciplinary Information Studies, University of Tokyo \\ 3-8-1 Komaba, Meguro-ku, Tokyo 153-8902 Japan \\ fukashiro@idaten.c.u-tokyo.ac.jp \\ ** Department of Life Sciences, University of Tokyo, Japan \\ 3-8-1 Komaba, Meguro-ku, Tokyo 153-8902 Japan \\ **** Computational Biomechanics Unit, RIKEN, Japan \\ 2-1 Hirosawa, Wako, Saitama 351-0198 Japan \\ [Received April 24, 2005 ; Accepted July 20, 2005]
}

\begin{abstract}
The fascicular and tendinous behavior of the human m. gastrocnemius medialis (MG) was measured using ultrasonography between vertical ankle- and drop-jumping in order to better understand how force is developed under dynamic conditions. These jumps employed movement at the ankle joint only, and the MG was defined as the agonist muscle of the jumps. Eight male subjects performed 1: Ankle plantarflexion jumping (AJ) from the standing position without countermovement, and 2: straight legged Drop jumping (DJ) from a height of $0.20 \mathrm{~m}$; both with maximal effort. Length, force and power output of the muscle-tendon complex (MTC), fascicles and tendinous structures were estimated from a video tape recorder (VTR)/force-plate system and ultrasonography. EMG of the triceps surae was simultaneously measured. Vertical displacements of the body center of gravity were $0.16 \mathrm{~m}$ in $\mathrm{AJ}$ and $0.33 \mathrm{~m}$ in DJ. There was no stretching phase of the fascicles during the take-off phase in both AJ and DJ. Physiological effects such as stretch reflex and potentiation did not appear to be present. Even in AJ, the fascicles of the MG shortened in the earlier phase of take-off compared to the MTC of MG. As a result, the elastic energy was stored (2.2 J) and reutilized (2.1 J: 47\% of total MTC work in latter phase) during the push-off phase. In DJ, on the other hand, much elastic energy was stored in the tendinous structures during dorsiflexion (7.6 J) and plantarflexion (5.8 J: 75\%) phases. It was quantitatively indicated that there was a large advantage from elastic energy use in DJ compared to AJ. The MG fascicles operated within the optimal phase of the sarcomere force-length relationship in AJ and DJ. Also, the fascicles worked in the relatively low shortening velocity region of the instantaneous force-velocity relationship in $\mathrm{AJ}$ and $\mathrm{DJ}$.
\end{abstract}

Keywords: muscle-tendon complex, plantarflexion vertical jumping, gastrocnemius medialis, elastic energy, ultrasonography

[International Journal of Sport and Health Science Vol.3, 253-263, 2005]

\section{Introduction}

Elastic properties of the muscle-tendon complex (MTC) play an important role in enhancing human performance, especially in stretch-shortening cycle (SSC) movements (Fukashiro, et al., 1995; Komi, 2000). The role of elasticity has been extensively investigated in many kinds of human and animal movements using experimental methods (Cavagna, et al., 1968; Edman, et al., 1982; Ettema, et al., 1992). In the case of human studies, especially, the buckle-type and the optic-fiber-type transducers (Komi, 2000) have been used for measuring the tendon force during movements in vivo. For technical and ethical reasons, human studies have been limited in their ability to clearly observe the phenomenon of elasticity in vivo (Fukashiro, et al., 1995).

Simulation studies which have eliminated the problems associated with using living human subjects have investigated how elasticity of the MTC contributes to work enhancement by modeling the 
SSC (Bobbert, et al., 1986; Bobbert, et al., 1987; Nagano, et al., 2003; Zajac, et al., 1984). These simulations have been seen as a powerful tool for estimating the contribution of elasticity during human movements. However, the constants of the elastic properties of the MTC, which are the most important parameters for determining the contribution of elasticity, were the values estimated based on either mechanical models, experimental animals, or human cadaver specimens (Bobbert, et al., 1986; Bobbert, et al., 1987; Lieber, et al., 1991). When mechanical values obtained from human subjects in vivo are compared to those estimated values, deviations are observed (Fukashiro, et al., 1995; Fukashiro, et al., 1995; Kurokawa, et al., 2003). It is important therefore, that the mechanical parameters of the human MTC be measured directly in vivo.

To examine the role of human elastic properties of the MTC, the comparative study of jumping with and without counter-movement has been used previously as the typical experimental design (Bobbert, et al., 1996; Fukashiro, and Komi, 1987; Komi, 2000). This is because elastic energy is stored in the tendinous structures when stretched by large forces in the downward phase of the SSC, and can be reutilized in the following push off phase (Cavagna, et al., 1968; Ettema, et al., 1992; Fukashiro, et al., 1995; Ingen, et al., 1997). Conversely, since there is no negative phase nor subsequent tendon stretch in non-counter movements, utilization of elastic energy is limited to that stored from maintaining posture prior to, and during the jump phase. Thus, the difference in stored elastic energy between the conditions with and without counter-movement, i.e., SSC, can be compared (Komi, 1978; Komi, 2000).

Recently, the methodology for measuring the longitudinal architecture of the MTC, i.e., length and angle of fascicles and length of tendinous structures was developed in vivo using B-mode real time ultrasonography (Fukashiro, et al., 1995; Fukunaga, et al., 2001). The constants of elastic properties in live humans can be directly but noninvasively determined by this method. This methodology has been applied to many kinds of natural human movements. Kubo, et al. (2000) and Fukunaga, et al. (2001) investigated the behavior of human $\mathrm{m}$. gastrocnemius medialis (MG) during sub maximal SSC plantarflexion and during human normal walking, respectively. Furthermore, high intensity and rapid movement has also been examined by Kurokawa, et al. (2003) through the use of ultrasonography by measuring the behavior of the MG during squat and counter movement vertical jumping with maximal effort. They reported quantitatively that for the MG during both jumping movements the fascicles shortened and the tendinous structures were stretched during the first phase of take-off, and that the fascicles generated force quasi-isometrically although the MTC shortened rapidly during the last phase of take-off. This behavior has been called "catapult action" (Hof, et al. 1983). Kurokawa, et al.(2003) defined the agonist muscles in squat and counter-movement jumps as hip and knee extensors. Although they focused on the MG, unfortunately it is not the primary contributor muscle of those movement jumps (Fukashiro, and Komi, 1987). In order to accurately assess the individual roles of the tendinous structure and the muscle fascicles for force development in the MG, it is essential to select a movement that minimizes the contribution of other muscles as well as limiting the number of joints undergoing flexion or extension.

Our current research goal has been to quantitatively measure using ultrasonography the amount of elastic energy utilization during a dynamic and natural human movement in which the primary agonist is the calf muscle. In such a movement, we would be able to accurately measure the work and power output of the MG. Our hypothesis was that reused elastic energy would be greater within the tendinous structures during a stretch-shortening DJ compared to a fully concentric AJ.

\section{METHODS}

\subsection{Subjects and experimental protocol}

Eight healthy male subjects (Age: $23 \pm 5$ years old; Body height: $1.72 \pm 0.10 \mathrm{~m}$; Body mass: $68 \pm$ $11 \mathrm{~kg}$; mean $\pm \mathrm{SD}$ ) participated in this study with informed consent. This study was approved by the Ethics Committee of the University of Tokyo. Subjects were barefoot and asked to perform the following two vertical jumps with maximal efforts; 1 : Ankle plantarflexion jumping (AJ) from the standing position without countermovement, and 2: straight legged drop jumping (DJ) from a height of $0.20 \mathrm{~m}$. Each subject performed 3 trials for each condition with high reproducibility. The trial for each condition that produced the maximal vertical displacement was 
selected for further statistical analysis.

The role of knee and hip extensors during drop jumping can be significant when subjects perform full range of motion jumps (Bobbert, et al., 1987). However, in our study, subjects were explicitly told to maintain a straight knee position during both counterand non-counter movement jumps. Compliance was monitored by video tape and incorrect movements were rejected from the study. While we cannot completely ignore the possibility of hip and knee flexor influence, we are confident that the knee range of motion was not a significant factor. A description of the movement phases and how they were selected is found in Figure 3.

\subsection{Kinematic and kinetic data collection:}

Reflective markers were placed over the following landmarks on the body: the fifth metatarsophalangeal joint, the lateral malleolus, the lateral epicondyle of the knee, the tip of the trochanter major and the glenohumeral joint. Four body segments, i.e. feet, shanks, thighs and HAT (head, arms and trunk), as well as joint angles were defined according to these landmarks. Subjects were filmed from the right side with a digital high-speed video camera at 200 $\mathrm{Hz}$ (MEMRECAMc2s, NAC, Japan). Vertical and horizontal ground reaction forces, as well as the center of pressure under the feet, were measured with a force platform at $1 \mathrm{kHz}$ (Type 9281B, Kistler Instruments AG, Switzerland, Figure 1). Time synchronization between the force platform and video data was achieved with an electrical timing switch.

Positions of the landmarks were automatically digitized using a motion analyzer (Frame-DIAS, DKH, Japan). Digitized data were low-pass filtered at $8 \mathrm{~Hz}$ (bidirectional Butterworth filter; Winter, 1990). Net joint moments and power output around the ankle and the knee joints were calculated using inverse dynamics (Winter, 1990).

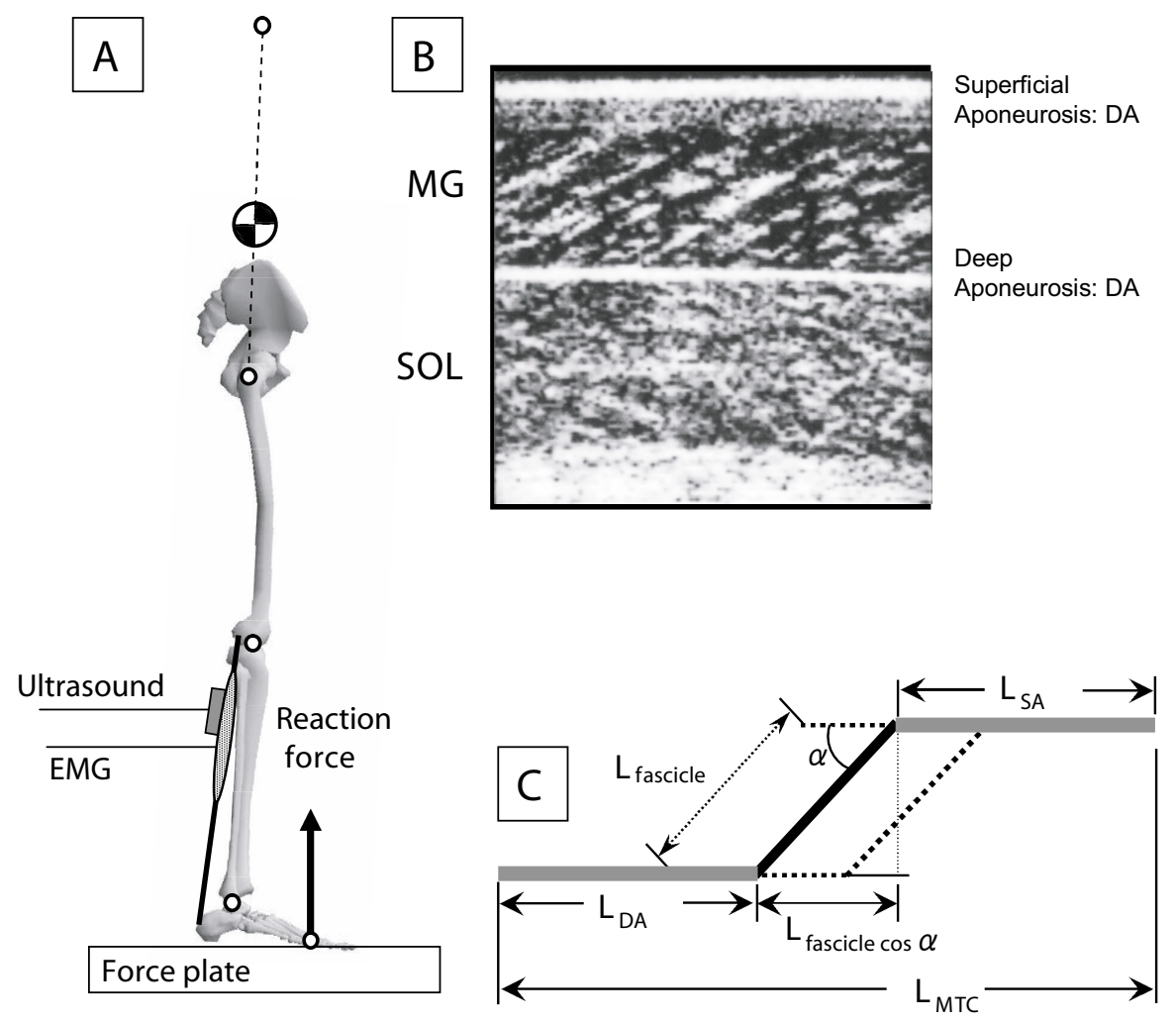

Figure 1 Schematic figure of this experiment.

A: Musculo-skeletal model. Kinematics and kinetics of the movement were measured by VTR and force-plate system, respectively. Joint moment was calculated by inverse dynamics. The ultrasound probe was placed on the MG. EMG was measured for each muscle. B: One ultrasound image of the MG. C: Calculation model of the MTC, fascicle and tendinous structures. LMTC: Length of MTC, LsA: Superficial aponeurosis, LDA: Deep aponeurosis, Lfascicle: Length of fascicle. 


\subsection{Ultrasonography}

A real-time B-mode ultrasonography unit (SSD-2000, Aloka, Japan) was used with an electronic linear array probe of $7.5 \mathrm{MHz}$ wave frequency (UST 5710-7.5, Aloka, Japan) to obtain longitudinal ultrasonic images of the MG (Kurokawa, et al., 2003). The field of view captured was $30 \mathrm{~mm}$ in length at a depth of $30 \mathrm{~mm}$ into the muscle. The probe was held in place by a specially designed brace that minimized unwanted movement. The accuracy of this device has been previously reported (Fukashiro, et al.,1995; Fukunaga, et al., 2001; Kubo, et al., 2000). During erect standing and jumping, the images of the MG were stored consecutively into the cine-memory of the ultrasonography at 40 $\mathrm{Hz}$ (Figure 1). Time synchronization was achieved by the electrical switch used for the force plate and camera synchronization.

Fascicle length was measured as the length from the superficial to the deep aponeurosis along the fascicles. Fascicle angle was obtained as the average value of two angles of the superficial and the deep aponeuroses. Reliability of fascicle length and angle measurements have been previously evaluated and validity of this methodology has been confirmed (Fukashiro, et al.,1995; Fukunaga, et al., 2001; Kubo, et al., 2000; Kurokawa, et al., 2003).

\subsection{Electromyography}

Surface electromyograms (EMG) were recorded from the MG, $\mathrm{m}$. gastrocnemius lateralis (LG), $\mathrm{m}$. soleus (SOL) and $\mathrm{m}$. tibialis anterior (TA) using bipolar Ag/Agcl electrodes (NEC Medical system, Japan) fixed longitudinally over the muscle belly. Raw EMG signals were high-pass filtered at $20 \mathrm{~Hz}$, low-pass filtered at $500 \mathrm{~Hz}$ (fourth order digital Butterworth filters); full-wave rectified and smoothed using a bidirectional digital low-pass Butterworth filter with a cut-off frequency of $2 \mathrm{~Hz}$ to yield Smoothed Rectified EMG (SREMG). SREMG was subsequently normalized (NSREMG) relative to the maximum value attained during each jump (Kurokawa, et al., 2003).

\subsection{Length of MTC, tendinous structures and moment arm}

Instantaneous length of the MTC of the MG
(Lмтс) was calculated from ankle and knee joint angles (Grieve, et al., 1978). Instantaneous length of the tendinous structures (Ltendon) was based on a geometrical MTC model reported by Allinger and Herzog, (1992).

$$
\text { Ltendon }=\text { LMTC }- \text { Lfascicle } \cdot \cos \alpha
$$

where Lfascicle, $\alpha$, and LMTC are fascicle length, fascicle angle, and MTC length, respectively. Instantaneous moment arm of the MG at the ankle joint was calculated by referring to Bobbert, et al., (1986).

\subsection{Fascicle and tendon force}

The Achilles tendon force was calculated from the plantar flexion moment and moment arm at the ankle joint. It was assumed that contribution of the MG relative to the whole Achilles tendon force was proportional to the physiological cross sectional area (PCSA) of the MG among all plantarflexors (15.4\%, Fukunaga, et al., 1996). Instantaneous tendon force generated by the MG (Ftendon MG) and the force of $M G$ fascicles (Ffascicles MG) were calculated as follows:

$$
\begin{aligned}
& \text { Ftendon } \mathrm{MG}=0.154 \cdot(\text { Mankle } \cdot d-1) \\
& \text { Ffascicles } \mathrm{MG}=\text { Ftendon } \mathrm{MG} \cdot \cos \alpha^{-1}
\end{aligned}
$$

where Mankle and $d$ represent plantarflexion moments and moment arm length at the ankle joint, respectively.

\subsection{Mechanical power and work of MTC}

We can evaluate quantitatively the mechanical properties of muscle and tendon during human movements in vivo using ultrasonography. Mechanical power produced by the MTC, fascicles and tendinous structures was calculated as the product of Ftendon MG and the velocity of the MTC and tendinous structures, respectively. For fascicles, Ffascicles MG was multiplied with the velocity of the fascicles to calculate the power. Negative and positive work performed by the MTC, fascicles and tendinous structures were calculated by numerically integrating the corresponding power values. The reported calculated values are for one leg only.

\subsection{Statistical procedure}

Mean time history of each variable (Joint angle, Joint moment, Joint power, MTC length, Fascicle length, Tendinous structures length, EMG, and 
Table 1 Average mechanical work done by the MTC, fascicles and tendinous structures of the MG during AJ and DJ.

\begin{tabular}{cllll}
\hline & AJ & DJ & AJ & DJ \\
\hline Phase & Upward-I & Downward & Upward-II & Upward \\
\hline MTC $(\mathrm{J})$ & $1.4 \pm 0.16$ & $-5.0 \pm 0.49^{* *}$ & $4.5 \pm 0.49$ & $7.7 \pm 0.58^{* *}$ \\
Fascicles $(\mathrm{J})$ & $3.6 \pm 0.55$ & $2.6 \pm 0.39$ & $2.4 \pm 0.36$ & $1.9 \pm 0.22$ \\
Tendinous $(\mathrm{J})$ & $-2.2 \pm 0.70$ & $-7.6 \pm 0.45^{* *}$ & $2.1 \pm 0.27$ & $5.8 \pm 0.61^{* *}$ \\
\hline
\end{tabular}

Values indicate means \pm S.E.M. ; $\mathrm{n} 8$

$* *: p<0.01$ significant difference between AJ in upward phase I and DJ in downward phase and between AJ in upward phase II and DJ upward phase.

Mechanical power) \pm standard error of means (S.E.M) was calculated for the whole group of subjects. Statistical evaluation of the average mechanical work done by the MTC, fascicles and tendinous structures of the MG during each phase was performed using a one-way analysis of variance (ANOVA) and t-tests for post hoc multiple comparisons where appropriate. The level of statistical significance was set at $P<0.05$. Significant differences are highlighted in Table 1.

\section{RESULTS}

Upward displacement of the body center of gravity of DJ $(0.33 \pm 0.04 \mathrm{~m})$ was significantly greater than that of $\mathrm{AJ}(0.16 \pm 0.02 \mathrm{~m})$ from the standing position. As shown in Figure 2, the peak of plantar flexion moment in DJ $(443 \mathrm{Nm})$ was much greater (about 2.2 times) than that of $\mathrm{AJ}(200 \mathrm{Nm})$, although the moment showed a similar pattern in AJ and DJ, i.e., a rapid increase followed by a rapid decrease. Ankle joint power generated by two legs in AJ showed only positive (peak: $963 \mathrm{~W}$ ) during upward phases I and II. Conversely, the ankle joint power in DJ attained a negative peak value of $-2174 \mathrm{~W}$ during the downward phase, but thereafter attained a positive peak value of $2793 \mathrm{~W}$ during the upward phase.

In AJ (Figure 3), the MTC length shortened slightly by $6 \mathrm{~mm}(1 \%$ relative to the length in the erect standing position) during the upward phase I. Fascicles, however, shortened largely by $13 \mathrm{~mm}$ (20\%). EMG activity of the MG, the LG and the SOL in AJ peaked around $-150 \mathrm{~ms}$, and as a result, the fascicles shortened largely during the upward phase I. The tendinous structures were slightly stretched ( $8 \mathrm{~mm}, 2 \%)$, storing elastic energy during this phase. The phase lag between the MTC and fascicles in the upward phase I was observed even in AJ. In the following upward phase II, the MTC (23 mm, $5 \%$ ), fascicles ( $8 \mathrm{~mm}, 12 \%$ ) and tendinous structures ( $15 \mathrm{~mm}, 4 \%)$ shortened, releasing prestored elastic energy. In DJ (Figure 3), on the other hand, the MTC was stretched by $17 \mathrm{~mm}$ (4\%) during the downward phase. The fascicles shortened by $9 \mathrm{~mm}(14 \%)$, and the tendinous structures were stretched by $6 \mathrm{~mm}(2 \%)$ with increasing muscle activity of the MG, LG and SOL, and with increasing plantarflexion moment. During phase II however, the MTC was shortened by $29 \mathrm{~mm}$ (7\%). During this upward phase, the fascicles shortened only slightly (quasi-isometric contraction) from 34 to $32 \mathrm{~mm}$ (5\%). Conversely, the tendinous structures shortened rapidly by $24 \mathrm{~mm}(7 \%)$.

Figure 4 shows the mechanical power of the MTC, fascicles and tendinous structures in AJ and DJ. In AJ, the power of the MTC (peak: $65 \mathrm{~W}$ ) and fascicles $(54 \mathrm{~W})$ was positive. However, the power of the tendinous structures was first negative (-27 $\mathrm{W})$ and then positive $(32 \mathrm{~W})$ in the upward phases I and II, respectively. Even in AJ, the elastic energy stored during upward phase I was reutilized during upward phase II. On the other hand, during the downward phase in DJ, the mechanical power of the fascicles was positive, and the tendinous structures were negative, with peak values of $51 \mathrm{~W}$ and -161 $\mathrm{W}$, respectively. Absolute peak mechanical power of the tendinous structures was 3.2 times higher than that of the fascicles. During the upward phase in DJ, mechanical power of the tendinous structures and the MTC became positive, thereafter reaching peak values of 126 and $151 \mathrm{~W}$, respectively. Mechanical power output of the fascicles was relatively low and 


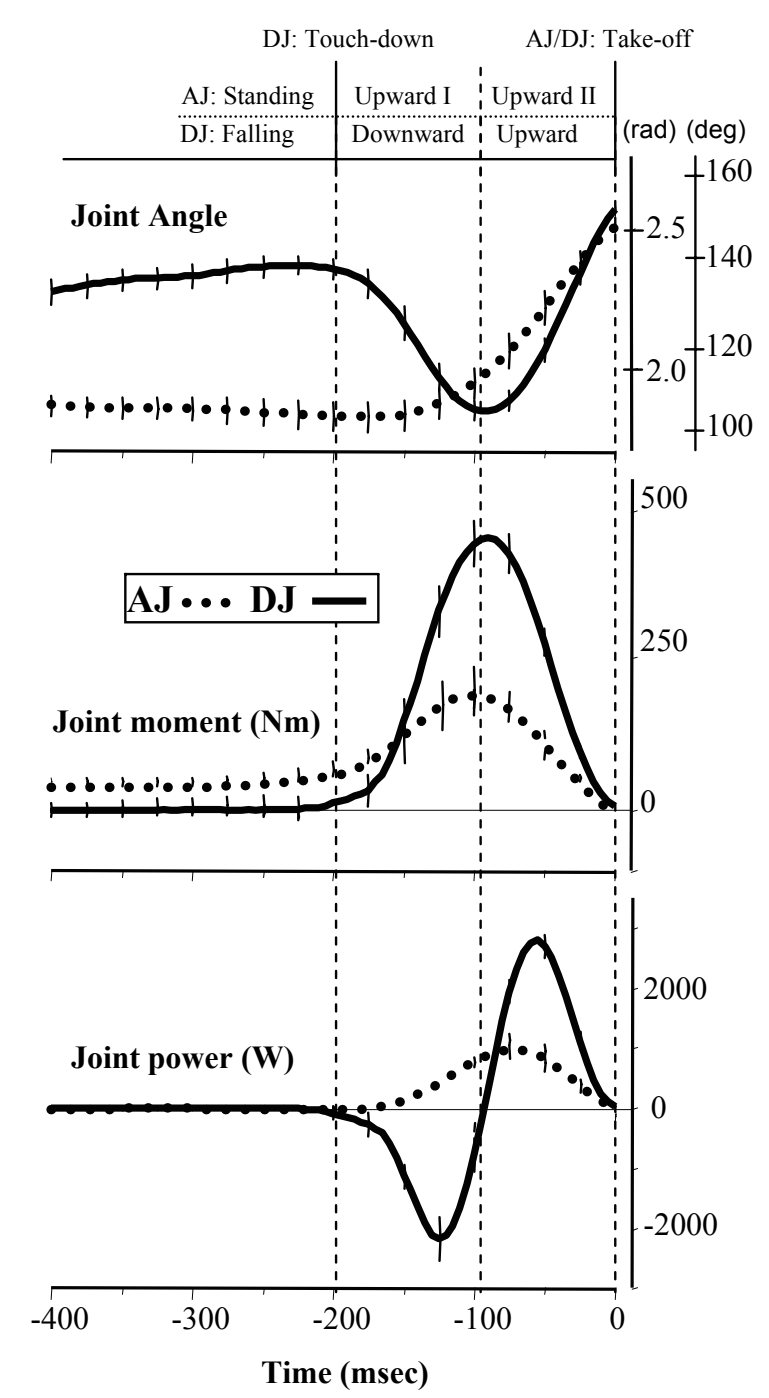

Figure 2 Mean time histories of joint angles, joint moments, and joint power at the ankle joint.

Positive joint moment around the ankle indicate plantarflexion moment. In figures 2 and 3, time is expressed relative to the instant of toe-off (Time $=0$ ). Thin vertical bars indicate S.E.M. $\mathrm{n}=8$ of subjects. Phase classification of these figures was defined as follows. The figures in AJ consisted conveniently into three phases: Standing, Upward I, and Upward II. The start of the upward phase I was defined as the onset of movement (-200 ms) and the start of upward phase II was defined as the peak of the ankle joint moment (-98 ms). DJ also consisted of three phases: Falling, Downward, and Upward. The downward phase was negative and occurred from touch-down $(-200 \mathrm{~ms})$ to maximal dorsiflexion $(-98 \mathrm{~ms})$. The upward phase was positive and was defined as the period from the end of the downward phase until take-off.

decreased to zero through this period.

Table 1 shows the mechanical work by each component and each phase in AJ and DJ. According to our results (Figure 2), there was a slight difference in the range of motion between the two movements within each phase. This may have had a slight impact
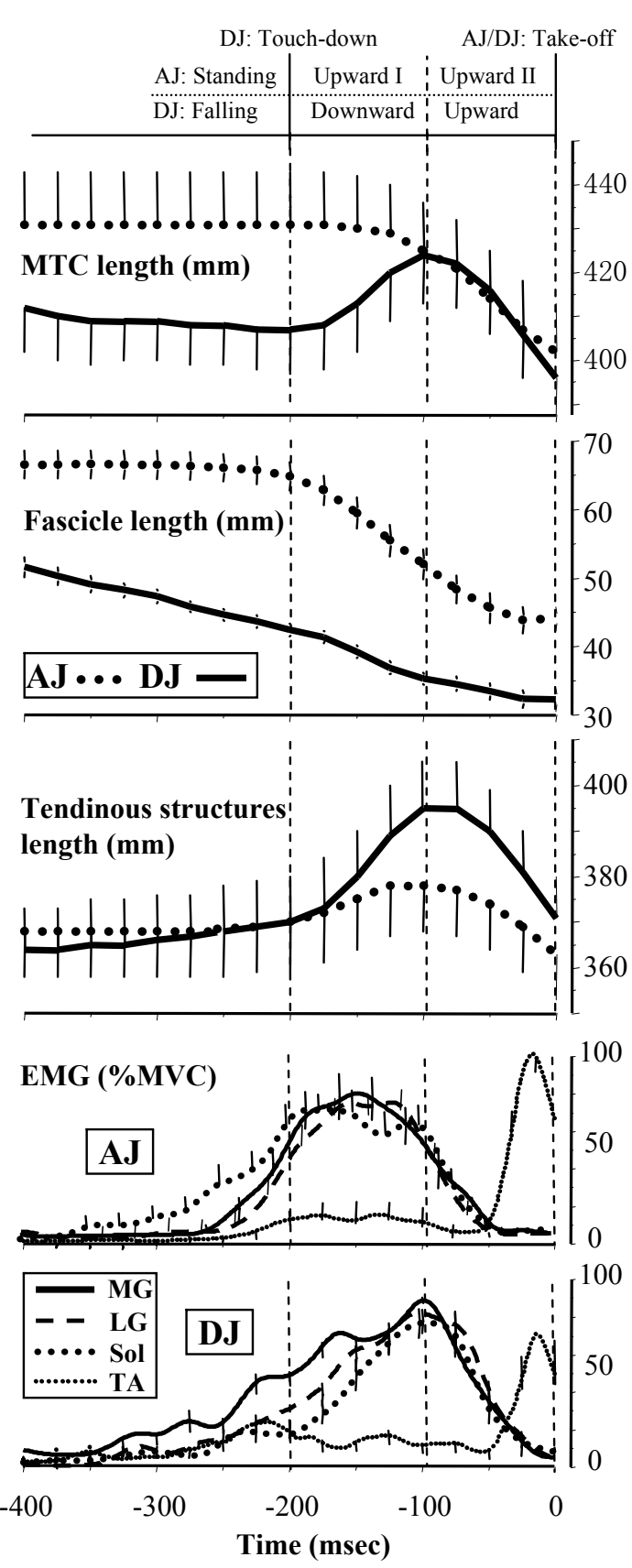

Figure 3 Mean time histories of MTC length, fascicle length, length of the tendinous structures, and normalized EMG activity from lower leg muscles during AJ and DJ. MG, gastrocnemius medialis; SOL, soleus; LG, gastrocnemius lateralis; TA, tibialis anterior.

on the calculated work and power values, but the fundamental differences in overall work and power would still be present. As there was no significant difference in fascicle work between AJ and DJ, both jumps were considered equal in terms of voluntary effort. During the upward phase I in AJ and 10 
the downward phase in DJ, the fascicles performed positive work of $3.6 \mathrm{~J}$ and $2.6 \mathrm{~J}$, and the tendinous structures performed negative work of $2.2 \mathrm{~J}$ and 7.6 $\mathrm{J}$, therefore the MTC performed $1.4 \mathrm{~J}$ of positive and $5.0 \mathrm{~J}$ of negative work, respectively. Also, during the upward phase II in AJ and the upward phase in DJ, of the total amount of mechanical work performed by the MTC (4.5J and 7.7J), the fascicles contributed $2.4 \mathrm{~J}(54 \%)$ and $1.9 \mathrm{~J}(25 \%)$, respectively; and the tendinous structures contributed $2.1 \mathrm{~J}(47 \%)$ and 5.8 $\mathrm{J}(75 \%)$, respectively. Mechanical work performed by the tendinous structures during the upward phase II in $\mathrm{AJ}$ and the upward phase in DJ were equivalent to $95 \%$ and $76 \%$ of the corresponding mechanical work performed during the upward phase I in AJ and the downward phase in DJ, respectively. These imply that $5 \%$ in $\mathrm{AJ}$ and $24 \%$ in DJ of the energy stored in the tendinous structures might be dissipated during plantarflexion.

\section{DISCUSSION}

The purpose of the present study was to compare qualitatively and quantitatively the behavior of the MTC of the MG during AJ and DJ using ultrasonography. Comparing the MG behavior between $\mathrm{AJ}$ and $\mathrm{DJ}$, the role of muscle and tendon interaction of the agonist muscle can be clearly evaluated with and without countermovement. These jumps were performed by utilizing only the ankle joint and by defining the MG as the agonist muscle of the jumps. Qualitative characteristics of the behavior of the MTC agreed well with previous experimental and simulation studies. In humans, on the other hand, it has been shown that the contractile component (CC) of the triceps surae muscle mainly performed concentric work (shortening of CC) while the series elastic component (SEC) of the triceps surae muscle was stretched during human hopping and walking (Hof, et al., 1983; Norman, and komi, 1979; Voigt,et al., 1998). Zajac, et al. (1984) reported almost the same result on triceps surae behavior of the ankle plantarflexion jump without countermovement by using computer simulation. Furthermore, Fukunaga, et al.(2001), Kubo, et al. (2000) and Kurokawa, et al. (2003) indicated that the muscle fibers of the MG contracted in a quasi-isometric manner during SSC plantarflexion, walking, and squat/counter movement jumps, respectively. In previous studies (Fukunaga, et al., 2001; Hof, et al., 2002; Kubo, et al., 2000) subjects performed movements that were less dynamic than drop jumping, and so the influence of the tendinous structures may not have been maximized. Kawakami, et al. (2002) studied plantar flexion using a leg press machine to measure MTC work and power. In that experiment, the non-counter and counter movement motions were similar to each other. In our experiment, the subjects performed much more rapid contractions $(200 \mathrm{~ms})$ in the non-counter movement condition compared to Kawakami et al's study (600 ms). The mean peak angular velocity of our experiment was $875 \mathrm{deg} / \mathrm{s}$ compared to Kawakami et al.'s study (318 deg/s). However, the mean fascicle shortening velocity between our study and theirs was not great $(65 \mathrm{~mm} / \mathrm{s}$ versus $73 \mathrm{~mm} / \mathrm{s}$ ), which is an indication that the tendinous structures act as a force controller during DJ by undergoing much greater elongation during the initial phase of take-off and more significant elastic energy reutilization during the last phase of take-off. Kurokawa, et al. (2003) used maximal dynamic movements to measure the MTC of the MG, but the selected movements for that study were squat and counter-movement jumps where the primary agonist muscle was not the MG. Our study was conducted to balance the challenges of measuring a natural and intense movement, yet limiting the number of agonist muscles involved. We have shown that due to the dynamic nature of ankle drop-jumping, the elastic properties of the tendinous structures play a more significant role in generating MTC work and power compared to less dynamic movements.

In SSC studies, the following mechanisms have been mainly assumed to explain the enhancement of maximum force/work and the mechanical efficiency through making a countermovement; 1 : storage and reutilization of elastic energy (Komi, and Bosco, 1978; Komi, 2000), 2: potentiation of the contractile machinery (Cavagna, et al., 1968; Edman, et al., 1982; Ettema, et al., 1992), and 3: contribution of stretch reflex (Dieta, et al., 1978; Komi, and Gollhofer, 1997; Melvill, and Watt, 1971). In our study, we sought to measure the tendinous and fascicle length changes during ankle jumping. The possible role of stretch reflex and potentiation related to tendon behaviour was not examined in the present study and thus their contribution to the results cannot be evaluated.

One of the major advantages of countermovement motion is to attain greater joint moments at the start 
of push-off compared to non-countermovement. As a consequence, more MTC work during countermovement can be produced than in non-countermovement (Bobbert, et al., 1996; Ingen, et al., 1997). Furthermore, in the present study, we had the advantage of comparing quantitatively the amount of elastic energy stored and utilized in the tendinous structures during movement. Since there was no falling phase in AJ, the tendinous structure was stretched only by fascicle shortening. A large proportion of the mechanical work by the fascicles $(3.6 \mathrm{~J})$ was stored as elastic energy in the tendinous structures $(2.2 \mathrm{~J})$ in the upward phase I. In other words, of the total work done by the fascicles, a significant amount did not translate into shortening of the total MTC. In the following phase II, the tendinous structure actually contributed to the total MTC work (2.1 J, $46 \%$ ) through reutilized elastic energy. While the fascicle work was still the greatest contributor to total MTC work $(2.4 \mathrm{~J})$, its fractional contribution was greatly diminished (Figure 4). In DJ, on the other hand, the elastic energy stored in the tendinous structures ( $7.6 \mathrm{~J}$, area of ' $\mathrm{b}+\mathrm{c}$ ' in Figure 4) consisted of work performed by the fascicles ( 2.6
$\mathrm{J}$, area ' $\mathrm{a}$ ') and the mechanical energy of the subject from the onset of the movement until the end of the downward phase (5.0 J, area of ' $b$ '). From our calculations, $66 \%$ of the elastic energy stored in the tendinous structures was provided by the mechanical energy change of the subject (area ' $b$ '), while the remaining $34 \%$ originated from the positive work performed by the fascicles (area ' $c$ ') during the downward phase. In the following upward phase, $76 \%$ of the elastic energy stored in the tendinous structures $(5.8 \mathrm{~J})$ was reutilized, and this accounted for $75 \%$ of the work performed by the MTC (Figure 4). With regard to the reutilization of stored elastic energy, it is advantageous for fascicles to generate higher force under a quasi-isometric condition, and to maintain relatively higher muscle stiffness during the upward phase in DJ. As a result, in the latter half of the take-off phase, the contribution by elastic energy in DJ was 1.63 times greater than that in $\mathrm{AJ}(75 \%$ in DJ and $46 \%$ in $\mathrm{AJ}$ ).

The length of sarcomeres in vertebrate striated muscles influences the force development capability of the muscle. The muscle fibers operate at sarcomere lengths near the plateau of the theoretical

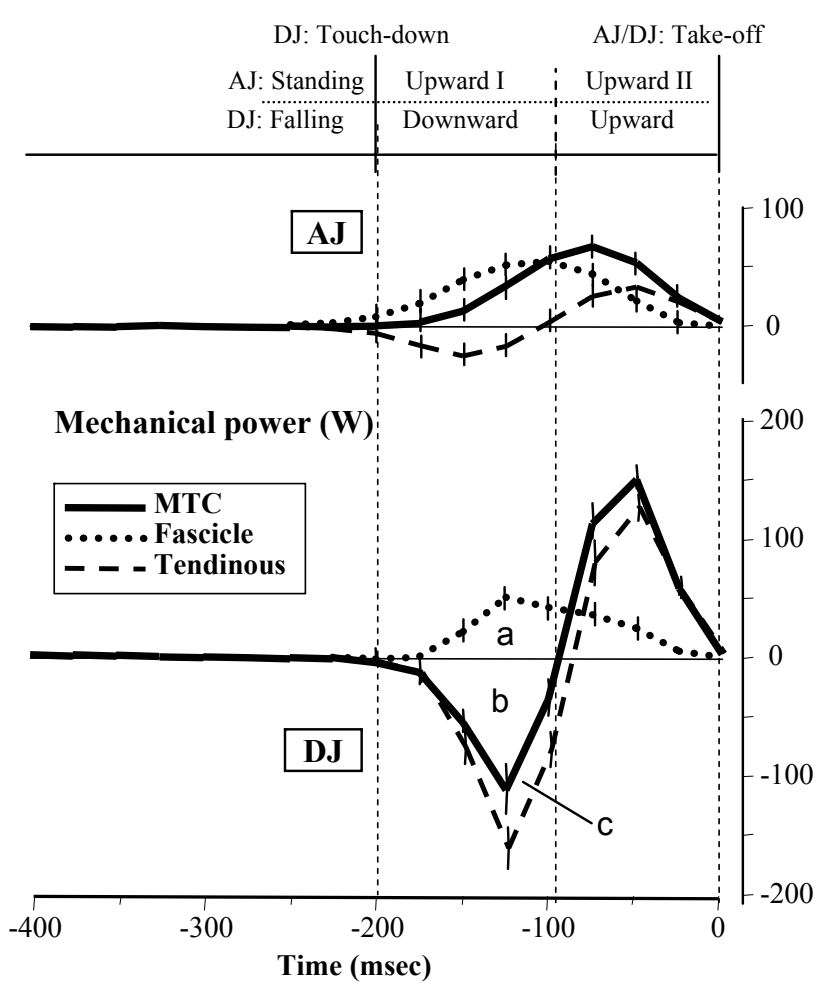

Figure 4 Mean time histories of mechanical power of the MTC, the fascicles and the tendinous structures of MG during AJ and DJ. Shortening of the MTC, fascicles and tendinous structures were defined as positive.

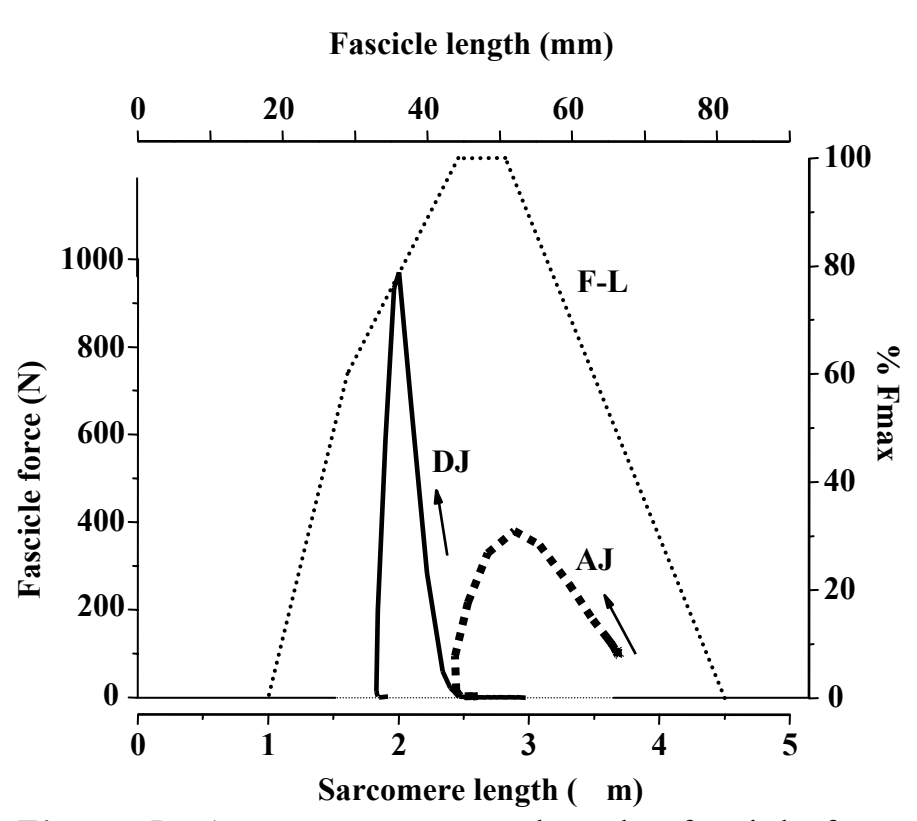

Figure 5 Average sarcomere length - fascicle force relationships during push-off and take-off phase in AJ and DJ.

Average sarcomere lengths during the push-off phase in each jumping motion have been estimated by dividing the fascicle length by number of sarcomeres $(3,27)$, and displayed with a force-length relationship (thin line) of human muscles derived from the data of Walker and Schrodt (33). 
force-length relationship, where maximum tension can be realized during human walking, hopping and daily activities (Cutts, 1989; Hof, et al., 1983; Hof, et al., 2002; Walker, and Schrodt, 1974). Figure 5 summarizes our hypothesized relationship between average sarcomere length and fascicle force during AJ and DJ. While we did not directly measure sarcomere length, and therefore cannot prove our conclusions, we think it reasonable to assume a correlation between the fascicle force-length relationship and the sarcomere force-length relationship. For example, a rapid shortening of the fascicles would be caused by a rapid sarcomere shortening, with a concomitant shift to the left of the force-length curve.

In AJ, we suggest that the MG operated firstly in the upper part of the descending limb, and secondly over the plateau phase of the sarcomere force-length relationship during the push-off. In DJ, on the other hand, MG operated over the plateau and the upper part of the ascending limb of the force-length relationship. Kawakami, et al. (2000) indicated that the MG and SOL operated over the plateau and upper part of the ascending/descending limbs of the force-length relationship during human isometric plantarflexion. When the MG and SOL contracted in an isometric manner with a maximal effort, the state of the system moved into the ascending limb of the force-length curve because of the fiber shortening. The results of our study indicate that the MG operated in the optimal area of the force-length relationship even in natural human movements (Hof, et al., 2002). The working range of the fascicles in DJ was very narrow and slightly shifted to left in comparison with AJ. Considering the results of Kawakami, et al. (2000), this condition in DJ is advantageous for muscle fibers to generate relatively high force, which is required in order to accelerate the body center of gravity during the latter half of the push-off phase, despite the slightly lower maximum force generation at the optimal length. When force generated by the fascicles peaked, sarcomeres operated at the length where they can generate about $30 \%$ in $\mathrm{AJ}$ and $80 \%$ in DJ of the force generated at the optimal sarcomere length. In the condition of natural, non-counter movements such as AJ, humans may not be able to generate maximal MTC force even if sarcomere length is optimal because the tendinous structures may not be fully utilized (Figure 5). The increase of vertical velocity of

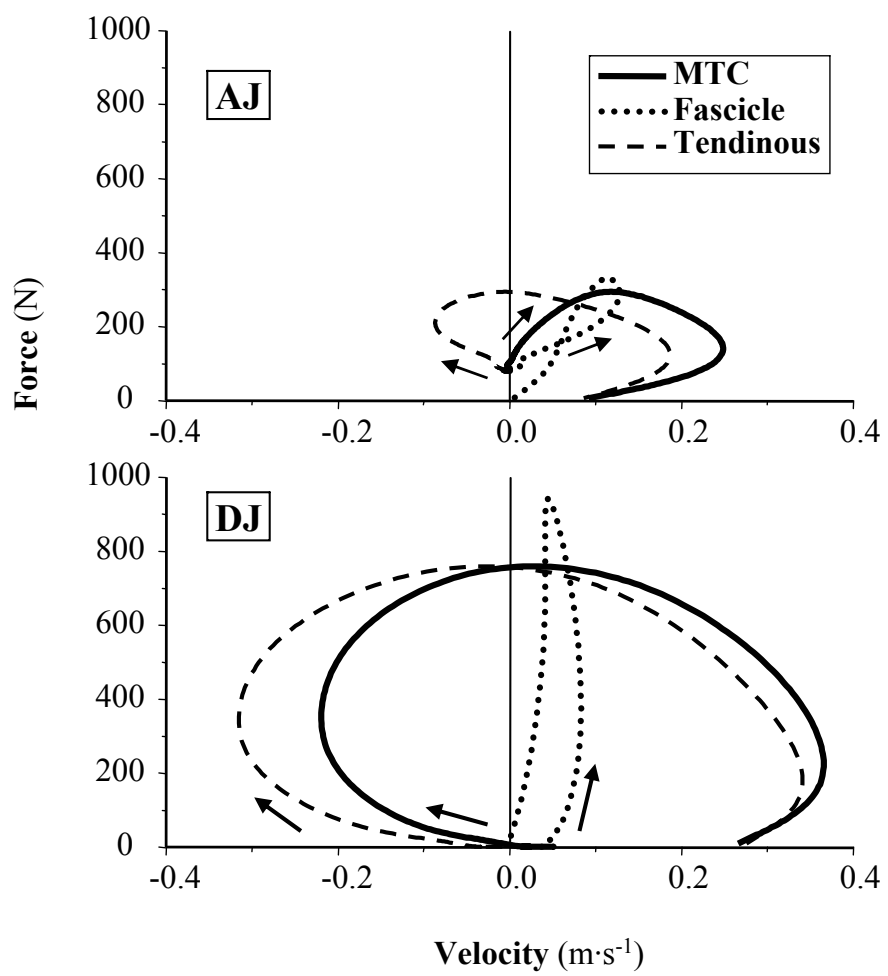

Figure 6 Instantaneous force-velocity relationships of the MTC, fascicles and tendinous structures of the MG in AJ and DJ.

the body center of gravity during the late push-off phase requires high mechanical power output from plantarflexors (Bobbert, et al., 1986). This suggests a combination of a relatively high shortening velocity of the MTC and a large force development in the mm. triceps surae. Figure 6 depicts the instantaneous force-velocity relationship of the MTC, fascicles and tendinous structures in $\mathrm{AJ}$ and DJ. In each jump, the fascicles worked in the relatively low shortening velocity region. Since the force generated by a muscle fiber decreases with increasing shortening velocity (force-velocity relationship), working in a quasi-isometric manner during the late push-off phase would enable the fascicles to generate relatively high force, especially in DJ. The quasi-isometric contraction of the fascicles is associated with the considerably rapid shortening of tendinous structures by which the shortening velocity of the fascicles could be lowered, despite the relatively higher shortening velocity of the MTC (Hof, et al., 2002). These data imply that the fascicles play a role in generating high tensional force (i.e., force generator), and the tendinous structures function in the manner that realizes high shortening velocity (i.e., velocity generator) to allow the MTC to generate high 
mechanical power during the late push-off phase in DJ. Moreover, it can be stated that rapid recoil of tendinous structures makes the muscle fibers behave in a quasi-isometric manner in this phase, where explosive power should be produced to accelerate the body center of gravity. Our results showed that there was a large advantage in the force-velocity relationship for generating higher power/work in DJ compared to that in AJ.

\section{ACKNOWLEDGMENT}

This research was supported by Ministry of Education, Culture, Sports, Science and Technology in Japan (No: 16300205).

\section{REFERENCES}

Allinger, T. \& Herzog, W. (1992). Calculated fiber length in cat gastrocnemius muscle during walking. Proc. NACOB II. The Second North American congress on Biomechanics, Chicago 81-82.

Bobbert, M.F., Huijing, P.A., \& van Ingen Schenau, G.J. (1986). An estimation of power output and work done by the human triceps surae MTC in jumping. J Biomech 19: 899-906.

Bobbert, M.F., Huijing, P.A., \& van Ingen Schenau, G.J. (1986). A model of the human triceps surae MTC applied to jumping. J Biomech 19: 887-898.

Bobbert, M.F., Huijing, P.A. \& van Ingen Schenau, G.J. (1987). Drop jumping. I. The influence of jumping technique on the biomechanics of jumping. Med Sci Sports Exerc. 19:332-338.

Bobbert, M.F., Gerritsen, K.G., Litjens, M.C., Van Soest, A.J. (1996). Why is countermovement jump height greater than squat jump height? Med Sci Sports Exerc, 26, 1012-1020.

Cavagna, G.A, Dusman, B., \& Margaria, R. (1978). Positive work done by a previously stretched muscle. J Appl Physiol 24: 21-32.

Cutts, A. (1989). Sarcomere length changes in muscles of the human thigh during walking. J Anat 166: 77-84.

Dietz, V., Schmidtbleicher, S., \& Noth, J. (1978). Neuronal mechanisms of human locomotion. J Physiol 238: 139-155.

Edman, K.A.P., Elzinga, G., \& Noble, M.I.M. (1982). Residual force enhancement after stretch of contracting frog single muscle fibers. J Gen Physiol 80: 769-784.

Ettema, G.J.C., Huijing, P.A., \& de Haan, A. (1992). The potentiating effect of prestretch on the contractile performance of rat gastrocnemius medialis muscle during subsequent shortening and isometric contractions. J Exp Biol 165: 121-136.

Fukashiro, S. \& Komi, P.V. (1987). Joint moment and mechanical power flow of the lower limb during vertical jump. Int $J$ Sports Med 8(suppl.):15-21.

Fukashiro, S., Ito, M., Ichinose, Y., Kawakami, Y., \& Fukunaga, T. (1995). Ultrasonography gives directly but noninvasively elastic characteristics of human tendon in vivo. Eur J Appl Physiol 71: 555-557.

Fukashiro, S., Komi, P.V., Jarvinen, M., \& Miyashita, M. (1995). In vivo Achilles tendon loading during jumping in humans. Eur J Appl Physiol 71:453-458.

Fukashiro, S., Noda, M., \& Shibayama, A. (2001). In vivo determination of muscle viscoelasticity in the human leg.
Acta Physiol Scand 172:241-248.

Fukunaga, T., Kubo, K., Kawakami, Y., Fukashiro, S., Kanehisa, H., \& Maganaris, C.N. (2001). In vivo behavior of human muscle tendon during walking. Proc R Soc Lond B Biol Sci B268: 1-5.

Fukunaga, T., Roy, R.R., Shellock, F.G., Hodgson, J.A., \& Edgerton, V.R. (1996). Specific tension of human plantarflexiors and dorsiflexors. J Appl Physiol 80: 158-165.

Grieve, D.W., Pheasant, S., \& Cavanagh, P.R. (1978). Prediction of gastrocnemius length from knee and ankle joint posture, In: Biomechanics VI-A, International Series on Biomechanics, edited by E. Asmussen, and K. Jorgensen. Baltimore: University Park Press, p.405-412.

Hof, A.L., Geelen, B.A., \& van den Berg, J. (1983). Calf muscle moment, work and efficiency in level walking: Role of series elasticity. J Biomech 16: 523-537.

Hof, A.L., Van Zandwijk, J.P. \& Bobbert, M.F. (2002). Mechanics of human triceps surae in walking, running and jumping. Acta Physiologica Scandinavica, 174, 17-30.

Ingen Schenau, G.J. van, Bobbert, M.F., \& de Haan. (1997). Does elastic energy enhance work and efficiency in the stretch-shorten cycle? Journal of Applied Biomechanics, 13, 389-415.

Kawakami, Y., Muraoka, T., Ito, S., Kanehisa, H. \& Fukunaga, T. (2002). In vivo muscle fibre behaviour during counter-movement exercise in humans reveals a significant role for tendon elasticity. J Physiol. 540:635-46.

Kawakami, Y., Kumagai, K., Huijung, P.A., Hijikata, T., \& Fukunaga, T. (2000). The length-force characteristics of human gastrocnemius and soleus muscle in vivo. In: Skeletal Muscle Mechanics: From Mechanisms to Function, edited by Herzog W: John Wiley and Sons, p. 327-341.

Komi, P.V. \& Bosco, C. (1978). Utilization of stored elastic energy in leg extensor muscle by men and women. Med Sci Sports 10: 261-265.

Komi, P.V. \& Gollhofer, A. (1997). Stretch reflex can have an important role in force enhancement during SSC exercise. Journal of Applied Biomechanics, 13: 451-460.

Komi, P.V. (2000). Stretch-shortening cycle: a powerful model to study normal and fatigued muscle. J Biomech 33 : 1197-1206.

Kubo, K., Kanehisa, H., Takeshita, D., Kawakami, Y., Fukashiro, S., \& Fukunaga, T. (2000). In vivo dynamics of human medial gastrocnemius MTC during stretch-shortening cycle exercise. Acta Physiol Scand 170: 127-135.

Kurokawa, S., Fukunaga, T., Nagano, A., \& Fukashiro, S. (2003). Interaction between fascicles and tendinous structures during counter movement jumping investigated in vivo. J Appl Physiol 95:2306-2314.

Lieber, R.L., Leonard, M.E., Brown, C.G., \& Trestik, C.L. (1991). Frog semitendinosis tendon load-strain and stress-strain properties during passive loading. Am J Physiol (Cell Physiol 30) 261: C86-C92.

Melvill Jones, G., \& Watt, D.G.D. (1971). Observation on the control of stepping and hopping movements in man. J Physiol (Lond) 219: 702-727.

Nagano, A., Komura, T., \& Fukashiro, S. (2003). Contribution of series elasticity in human cyclic heel-raise exercise. $J$ Appl Biomech 19, 340-352.

Norman, R.W. \& Komi, P.V. (1979). Electromechanical delay in skeletal muscle under normal movement conditions. Acta Physiol Scand 106: 241-248. 
Voigt, M., Dyher-Poulesen, P., \& Simonsen, E.B. (1998). Modulation of short latency stretch reflexes during human hopping. Acta Physiol Scand 163: 181-194.

Walker, S.M. \& Schrodt, G.R.I. (1974). Segment length and thin filament period in skeletal muscle fibers of the Rhesus monkey and the human. Anat Rec 178: 63-82.

Winter, D.A. (1990). Kinetics: Forces and Moments of force, In: Biomechanics and motor control of human movement (2nd Edition), edited by DA Winter. New York: John Wiley and Sons, p.75-102.

Zajac, F.E., Wicke, R.W., \& Levine, W.S. (1984). Dependence of jumping performance on muscle properties when humans use only calf muscles for propulsion. J Biomech 17, 513-523.

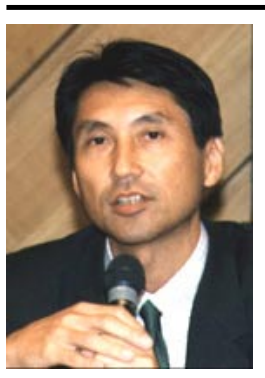

Name:

Senshi Fukashiro

\section{Affiliation:}

Graduate School of Interdisciplinary Information Studies, University of Tokyo

\section{Address:}

3-8-1 Komaba, Meguro, Tokyo 153-8902, Japan

Brief Biographical History:

1993 PhD, University of Tokyo

1993- Executive Council of Japanese Society of Biomechanics 1999-2005 Executive Council of International Society of Biomechanics

1998-2004 Executive Council of Japan Society of Physical Education, Health and Sport Sciences

2002- President of Tokyo branch of Japan Society of Physical Education, Health and Sport Sciences

2002-2004 Associate Editor of Journal of Japan Society of Physical Education, Health and Sport Sciences

2003-Associate Editor of International Journal of Sport and Health Science

\section{Main Works:}

- S. Fukashiro (1990): Science of Jumping. Taishukan-shoten, Tokyo.

- S. Fukashiro, S. Sakurai, Y. Hirano and M. Ae (2000): Sport Biomechanics. Asakura-shoten, Tokyo.

- S. Fukashiro and A. Shibayama (2000): Handbook of Mathematics and Physics in Sports. Asakura-shoten, Tokyo.

- S. Fukashiro, M. Noda and A. Shibayama (2001): In vivo determination of muscle viscoelasticity in the human leg. Acta Physiol. Scand. 172:241-248.

- S, Fukashiro, T. Abe, A. Shibayama and W.F. Brechue(2002): Comparison of viscoelastic characteristics in triceps surae between Black and White athletes. Acta Physiol Scand. 175(3):183-187.

Membership in Learned Societies:

- Japanese Society of Biomechanics

- International Society of Biomechanics

- Japan Society of Physical Education, Health and Sport Sciences 